\title{
Development of a Low-Cost GSM-Bluetooth Home Automation System
}

\author{
Salihu Aliyu ${ }^{1}$, Abdulazeez Yusuf ${ }^{1}$, Umar Abdullahi ${ }^{1}$, Mustapha Hafiz ${ }^{2}$, and Lukman A. Ajao ${ }^{3}$ \\ ${ }^{1}$ Department of Telecommunication Engineering, Federal University of Technology, Minna, Niger State \\ ${ }^{2}$ Synchronous Logic Engineering Limited, Minna, Niger State \\ ${ }^{3}$ Department of Computer Engineering, Federal University of Technology, Minna, Niger State \\ E-mail: salihu.aliyu@ @utminna.edu.ng, yusufazeez28@gmail.com, umarabdullahi@futminna.edu.ng, \\ bola4micro@gmail.com, ajao.wale@ futminna.edu.ng.
}

Received: 07 March 2017; Accepted: 14 April 2017; Published: 08 August 2017

\begin{abstract}
In today's age of digital technology and intelligent systems, home automation has become one of the fastest developing technology in the world as more and more people begin to see the idea of remotely monitoring and controlling their home appliances more as a necessity rather than a luxury. This paper presents the design and development of a smart home system that allows control of home appliances using both Bluetooth and GSM technology. The use of multiple control mediums gives more robustness to the system as appliance control and monitoring is made cheaper and possible regardless of the distance from which control is being effected. The system is controlled using a dedicated android based application which ensures convenience and ease of use. In addition, it is equipped with a security feature which is activated when the user is away from home. This enables the user to detect intrusion while the user is away.
\end{abstract}

Index Terms-Android App, appliance control, Bluetooth technology, GSM technology, home automation, smart home.

\section{INTRODUCTION}

The advent of technology and digital systems has justified communication as the backbone for most recent innovations in various engineering disciplines. It is worth noting that in recent years the concept of owning a "smart home" or a fully automated home system has become one of the fastest developing technology in the world. There is an increasing need to have intelligent systems which can conveniently control and keep track of events around your home irrespective of your distance from home. This is being made possible as more researchers and engineers continue to break new ground and create endless possibilities in the areas of home automation and real time monitoring.

Considerable efforts and achievements have been recorded in the development and design of remotely controlled systems. The earliest works for this systems can be traced back to the turn of the century when telephone and electrical wiring were implemented in most modern homes. In more recent times the proliferation of telecommunication technologies has rechanneled developer's efforts towards the use wireless technologies in achieving the design of a smart home. The availability of multiple wireless technologies such as Bluetooth, GSM, Infrared, ZigBee, Wi-Fi and RFIDs has made the concept of "smart home" a commercially viable one.

This paper proposed the design and development of a GSM-Bluetooth based home automation system (HAS) with an accurate feedback mechanism, minimum latency and a user friendly control interfaces. The system design is intended to limit cost resulting from controlling the appliances. In addition to cost effectiveness, a secured and easily scalable system is proposed herewith. The Bluetooth technology would be employed when in close range while control via SMS would be used from remote locations. The essence of this is to mitigate cost and eliminate SMS charges that would have resulted from controlling the appliances using SMS while within proximity of the appliances. The design also include a security feature (PIR sensor) that can help detect intrusion in the home and send alerts to appropriate authorities while the user is away. A combination of Bluetooth and GSM modules, Atmega8 microcontroller, relay drivers, power supply unit, and PIR sensor were employed to actualize the design. This paper presents a prototype design which is portable, easy to install, reasonably priced and can easily be used by electronics consumers. The rest of this paper is organized as follows: section II presents review of previous work in home automation systems, while section III presents the design of the proposed solution. Sections IV and V present the system implementation and evaluation respectively, whereas the paper is concluded in section VI.

\section{RELATED WORKS}

There have been numerous research works in the literature where the issue of automating home have been addressed using different communication technologies. Previous works can be categorized based on communication medium used as: internet, DTMF signaling, GSM network, Bluetooth, and ZigBee 
technology. In what follows, a review of home automation research using these communication mediums is hereby presented.

\section{A. Home Automation using Bluetooth}

Bluetooth is a wireless technology for transferring data between two devices that are in close proximity; it is most suitable for short timed communications. Bluetooth communication technology does not require line of sight between the two communicating nodes. Thus, making it a viable option for home appliance control over the traditional remote control. In [1], the authors developed 'PICONET', which basically is a network of Bluetooth devices. The authors made use of a master-slave connection between several devices. The control or central Bluetooth module is attached to a microcontroller chip which serves as the master device while the slaves are the modules attached to the various appliances. An android phone was used as the mobile unit and commands were issued via the Bluetooth application on it. The microcontroller was set in a polling status and constantly checks any input command every 500 millisecond from the Android phone application. The use of master-slave structure ensured that existing structures are not tampered with and scalability becomes easy. However, control from remote location and cost of implementing this on a large scale is still a huge limitation. A low cost, flexible and secure cell phone based home automation system was discussed in [2]. The home appliances were connected through relay to the I/O port of a stand-alone Arduino board for the control of home environment devices. The system design was enhanced with the incorporation of security assistance using password technique to prevent unauthorized user to the smart home control system. Therefore, the mobile phone operation was script in python programming language which allows it to function on any platform of Symbian OS. The system is capable of switching ON/OFF appliances without the need for internet connectivity. The performance range in a concrete building was reported to be greater than $50 \mathrm{~m}$, and in an open area to be $100 \mathrm{~m}$. [3] presented a Bluetooth based smart home design in which an android application was used as the control interface. The application allowed the user to control devices by simply pushing ON/OFF buttons on the application interface. On activation, the application creates a session with the Bluetooth module at the control unit, formats the commands as required and sends it via the mobile phones Bluetooth interface. On reception of this commands, the Microcontroller processes the data and sends necessary control signals to the connected relays which in turn switches the state of the appliances as required. The system is user friendly and more devices can easily be integrated. However, the limited range of Bluetooth still remains a major drawback as control from remote locations is impossible.

\section{B. Home Automation using ZigBee}

Some other authors have considered using the ZigBee wireless technology for home automation system. ZigBee is a radio frequency (RF) communications standard based on IEEE 802.15.4 [4]. A ZigBee-based home automation system was put forward in [4] to address the shortcoming of connectivity and interoperability of home appliances. ZigBee wireless technology and Wi-Fi network for home automation was integrated through a gateway, virtual home system for control and monitoring home devices using remote control system. The implementation of home gateway was to provide network interoperability, flexibility of user interface, simple connectivity and to permit remote access to the home system environment. In addition, the virtual gateway was implemented to cater for security and safety requirements. The system was proposed to cater for four home appliances like switching of light, valve warmer, safety sensor and remote control.

In [5], the authors developed a security control and monitoring system based on ZigBee technology. The system comprises of ZigBee modules and ZigBee end devices. The system was designed to control electrical doors and gas system via a switching circuit which is also equipped with a ZigBee node. Whenever intrusion is detected, the home owner is notified via SMS. The owner can send a command to shut all doors and activate the gas system depending on the need. The proposed system has a basic advantage over Bluetooth technology based systems in that it has a far better range and generally ZigBee modules consume less power compared to Bluetooth modules. However, control is still limited by range. In [6], [7], a voice control system was developed for a ZigBee based home automation system, while [8] proposed the development of a wireless smart home security system based on ZigBee and GPRS technology. It consists of a host control system and other sub functional modules. The host control system was made up of the GPRS module, ZigBee module and PIR sensor. The Sub functional modules consists of the data acquisition module, centralized switch and ZigBee module. It utilized the master-slave structure kind of communication between the centralized ZigBee module and the sub functional modules. The host sends command messages via GPRS mobile phone to the master slave or host control center which drives the control of appliances connected to it. An Energy efficient HAS was proposed in [9]. The system consists of network of sensors and appliance actuators to get information and control house environment respectively. An Arduino microcontroller was used as a central controller for communicating with an Android application and user interface. The proposed system made use of wireless ZigBee and wired X10 technologies.

\section{Home Automation using GSM Network}

Short Message Service (SMS) allows text messages to be sent and received using mobile phones. The text can comprise words or numbers or an alphanumeric combination. SMS was the most widely used data application, with an estimated 3.5 billion active users, or about $80 \%$ of all mobile phone subscribers at the end of 2010. The ease of use and popularity of this communication system explains why it is widely 
deployed in smart home development.

Design of Home Automation System (HAS) for monitoring and controlling of household appliances in remote locations can be achieved through various communications technologies such as wireless LAN technology, dial-up modems, Bluetooth, Zig-Bee, satellite communication and cellular networks. Among the options of these technologies, cellular network has attracted attention of researchers due its wide coverage area, cost effectiveness compare to other mobile communications and it is a highly secured network. The cellular network mostly employed is the GSM (Global System for Mobile communication) standard. A design and implementation of a machine-2-machine system was proposed in [10] using GSM network. The author concluded that the system can be implemented using many technologies such as Dual Tone Multi Frequency (DTMF), Short Message Services (SMS), and General Packet Radio Services (GPRS). The designer is to choose the most suitable technology for any particular application. The authors in [11] proposed the design of a basic smart home system which allows users to control home appliances via text messaging over a given GSM network. The design was targeted at providing a simple and easy to control smart home system that is very affordable. The mobile unit (user) sends control commands to the mobile device stationed at the control center. The microcontroller then extracts this commands from the mobile device, processes the commands and sends control signals to the relay drivers which in turn drives the relays thereby switching the connected devices ON/OFF. The user then gets a feedback message showing the commands executed and current state of the devices. The system has the advantage of simplicity and ease of implementation but the drawbacks are that the system is not robust and one might encounter much difficulty in integrating new devices into the system. In addition, frequent use of system indicates higher cost over time. Another approach was proposed in [12], the authors developed a framework for home automation using $\mathrm{J} 2 \mathrm{EE} / \mathrm{J} 2 \mathrm{ME}$ platform. In this system, the control of household is done with the aid of mobile phones. The paper analyses the design plan of both the server terminal and the mobile phone user. Unified Model Language (UML) was also used in the modelling of HAS. However, this approach requires internet service and also depends on the battery of the mobile phone to be able to control the devices. Therefore, the system may not be reliable in the absence of internet and when the battery of the mobile phone is drained out. In [13], another approach for control of household appliances using cell phone with the aid of GSM technology was developed. The system uses SMS to control household appliances from far distance. It is more adaptable and cost effective and also provides ubiquitous access especially where internet facilities are not available. Other similar works have also been presented in [14]-[19].

\section{Home Automation using DTMF Signaling}

Dual-tone multi-frequency signaling (DTMF) is an in- band telecommunication signaling system using the voice-frequency band over telephone lines between telephone equipment, other communications devices, and switching centers. The tones produced when dialing on the keypad of mobile phone could be used to represent the digits since a separate tone is used for each digit. In [20], the authors proposed a remotely operated mobile phone controlled home appliances. It makes use of the DTMF, two mobile phones, DTMF decoder, and an atmega8 microcontroller. One mobile phone was used as the remote device via which the user can send control commands, while the second mobile phone as the receiving device. This approach is expensive as it requires two phones, not user friendly; users will need to memorize control commands to operate a given device. In addition, a phone call must always be established with the mobile phone at home which introduces delay to the system. The authors in [21] presented an home automation system using DTMF with a noise reduction features using Goertzel DFT estimation to eliminate the noise in the system. Similar work using the DTMF approach was presented in [22].

\section{E. Internet-Based Home Automation}

The internet became a commonplace in the twentieth century and the basic advantage it holds over all other forms of wireless communication is that it is unlimited by distance or location and it is universal. The internet has formed the backbone for a new trend in home automation popularly referred to as the Internet of Things (IoT). Since internet connectivity is becoming everywhere which also help in driving the IoT technology, an IPbased home automation system was proposed in [23]. In order to simplify the cost and complexity of IP-based system, the authors developed a hardware/software framework called IPAcBox which allows home/office automation systems to be accessed and controlled through a direct connection right out of the box. The system also works in the event of dynamic IP configurations. However, the system is expensive to implement compare to other GSM based approach. In [24], the authors came up with an ubiquitous COAP-based home automation system using time synchronization for the development of low power sensor modules with smart home control system. COAP protocol was standardized recently for the sensor network application to operate between M2M in the smart home appliances. The authors proposed that COAP scheme can be applied to both IP-based and nonIP-based home automation gadgets. This lightweight Constrained Application Protocol is a web-based application-level protocol that supports IEEE 802.15.4 low level standard for sensor networks and supports (RESTful) architecture. The time synchronization scheme presented in their paper was aimed at minimizing both resource usage and network overhead that exist on the sensor nodes and home gateway, in order to achieve better time accuracy in comparison with existing design.

An Internet Based Wireless Home Automation System for Multifunctional Devices was discussed in [25]. The aim of the paper was to design and implement a central 
point control home automation system, low-cost, flexible and secure internet based system. The authors suggested a framework for a communication protocol that operates between several devices available in the home automation than common switching techniques. The system connections between the different nodes (i.e., the user interface, the database and the web based connections) were modeled in unified modeled language (UML). In the hardware system design, PIC16F877 microcontroller was used with RF wireless technology to transmit signal via MAX232 serial port to the web server. However, the system was demonstrated to control only switching of light in the home environment. An internet based home automated system was proposed in [26], [27] in which a smart home developed on an Arduino platform was controlled and monitored via Email over the internet. A user-friendly HAS based on 3D virtual world was proposed in [28]. The paper proposed design of a realistic $3 \mathrm{D}$ view interface as an improvement on the existing user interface. In addition, a home server was used as a controller for home devices. With this 3D virtual World, a user can control and monitor home devices via a userfriendly interface that works both intuitively and realistically anywhere and anytime through the Internet. High cost and availability of internet still remains the shortcomings of the system. A review of IoT technologies, architecture, challenges, applications and future trend have been presented in [29].

\section{F. Home Automation using Multiple Technologies}

The most important factors that should influence the design of a smart home system include the scalability of the system, security, ease of integrating new appliances and devices into the existing design, ease of use or a user friendly control interface and cost effectiveness of the overall system. In recent designs, researchers are faced with a tradeoff between most of this factors as the pursuance of one of these factors may limit the implementation of another. To implement such a system that integrates all these factors, some researchers have proposed HAS that utilizes two or more of the available wireless technologies. In [30], the authors combined both GSM and Bluetooth technology for monitoring and control of home appliances and sensors. A combination of ZigBee and GSM technology was proposed in [8]. The authors in [31] combined four different technologies; Bluetooth, GSM, Wi-Fi and DTMF to achieve a low cost, flexible, and standalone home automation system. Though the system can provide users with different means of controlling the home appliances, however, the use of too many communication technologies can introduce complexity in the system thus leading to high cost of production.

\section{G. Features of the Proposed System}

Several novel approaches have been proposed in the literature for home automation system, however, there still exits some limitations ranging from high cost of implementation to complex control mechanism. To minimize the various drawbacks evident in all the aforementioned systems, the system proposed herewith implements a number of features and measures to achieve a low cost smart home system for consumers. The proposed system has the following features:

> The system utilizes both GSM and Bluetooth wireless technologies for remote users and nearby users respectively.

$>$ The system uses an android application to provide a friendly user interface for easy control of appliances.

$>$ It also has support for non-users of smart phone.

$>$ A feedback system using current sensors to check if current actually flows through the activated appliance(s).

$>$ A security features using PIR sensor to detect intrusion while the user is away from home.

$>$ Home electric power utility status request; this enables users to know the status of power from a remote location. This feature is very important, especially for developing countries where there is incessant power cut, as it allows users to plan for alternative power source before arriving home.

All communications to the proposed HAS are checked for security and proper message format before any request from the users is serviced.

\section{SYSTEM DESIGN}

This section describes the conceptual design of the proposed low-cost smart home system. The system block diagram is as shown in Fig. 1. As shown in the figure, remote users can control the devices through the GSM network while nearby users make use of Bluetooth network.

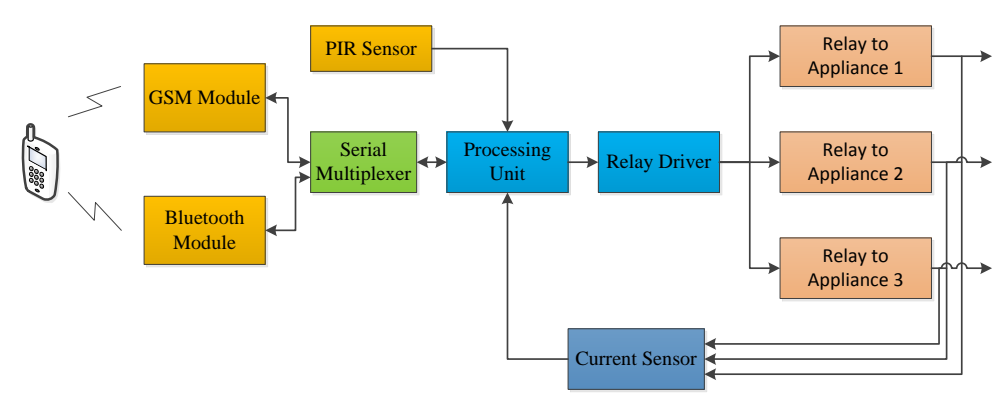

Fig.1. Functional block diagram of the proposed system 
The design also includes a security feature using the PIR (Passive Infrared) sensor to detect intrusion whenever the user is away. In this section a thorough discussion of the design units are presented.

\section{A. Bluetooth Technology}

For nearby users, the home appliances can be controlled via Bluetooth. This was achieved by establishing a connection between the users' phone, with Bluetooth capability, with a Bluetooth module connected to the control unit. The Bluetooth module is as shown in Fig. 2.

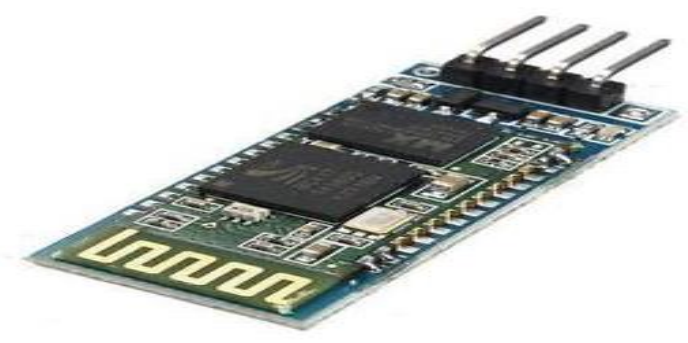

Fig.2. Snapshot of the Bluetooth module

The Bluetooth module has 4 pins, which are Vcc, ground, $\mathrm{Rx}$, and $\mathrm{Tx} . \mathrm{Rx}$ is the receiver signal pin and $\mathrm{Tx}$ is the transmitter signal pin. The Bluetooth $\mathrm{Rx}$ and $\mathrm{Tx}$ were connected to an Atmega16 microcontroller Rx and Tx respectively. However, since the Atmega16 microcontroller has only one set of $\mathrm{Tx}$ and $\mathrm{Rx}$, and a second serial device (GSM module) will need to be connected, a serial multiplexer was used to connect the Bluetooth and GSM module as will be discussed in this section.

\section{B. GSM Technology}

In the proposed system, the SMS (Short Message Service) feature of GSM technology was used to achieve control from a remote location. SMS can make all communication in short. It has gained widespread application in home appliance control. It allows users to control home appliances from a remote location by sending a short command. Unlike the work in [31] and some other works, users are not required to memorize control commands in the proposed system. All required commands have been embedded in a friendly user application (App) developed for Android OS. However, for the purpose of flexibility, users without smart phone can also control the appliances by using SMS to send the required commands.

The SMS sending and receiving functions were achieved using a SIM800L GSM module. The module is provided with a SIM (Subscriber Identity Module) which enables it to send and receive SMS. It can work with SIM from any of the available network providers. The module is configured using standard AT commands and is capable of performing operations of conventional mobile phones. The module used here also supports an "extended AT command set". It has current consumption of $250 \mathrm{~mA}$ during normal operation. It also supports Voice, Fax/Data GPRS and integrated TCP/IP suite. It was connected to the microcontroller along with the Bluetooth module via a serial multiplexer.

\section{Serial Multiplexer}

The microcontroller used has one set serial port (i.e. Rx and Tx) and since we made use of two devices that uses serial communication, as such the $74 \mathrm{HC} 4052$ multiplexer is implored. The 74HCT4052 is a dual single-pole, quadthrow analog switch (2x SP4T) suitable for use in analog or digital 4:1 multiplexer/de-multiplexer applications. Each switch features four independent inputs/outputs (nY0, nY1, nY2 and nY3) and a common input/output (nZ). A digital enable input (INH) and two digital select inputs (S0 and $\mathrm{S} 1$ ) are common to both switches. When $\mathrm{E}$ is HIGH, the switches are turned off. The function table of the 74HC4052 is shown in Table 1, while the circuit connection is shown in Fig. 3.

Table 1. Function Table of 74HC4052

\begin{tabular}{llll}
\hline Inputs & & & Channel On \\
\hline Enable (INH) & $\mathrm{B}$ & $\mathrm{A}$ & $\mathrm{X} 0, \mathrm{X}$ \\
\hline $\mathbf{0}$ & 0 & 0 & $\mathrm{X} 1, \mathrm{X}$ \\
\hline $\mathbf{0}$ & 0 & 1 & $\mathrm{Y} 0, \mathrm{Y}$ \\
\hline $\mathbf{0}$ & 1 & 0 & $\mathrm{Y} 1, \mathrm{Y}$ \\
\hline $\mathbf{1}$ & 1 & 1 & None \\
\hline
\end{tabular}

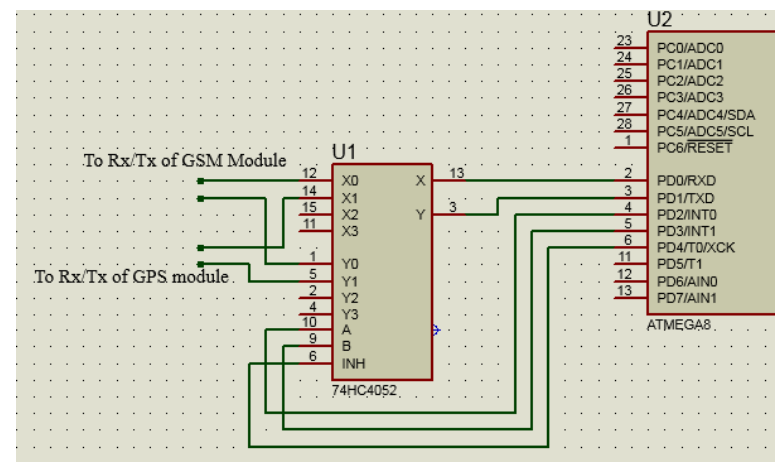

Fig.3. Serial Port Multiplexer (74HC4052) connection to Atmega8 microcontroller

Table 1 shows how the receiving port (Rx) and transmitting port (Tx) are selected using the selection pins $A$ and $B$. Pin $X$ goes to receiving (RXD) pin of the microcontroller, while pin $\mathrm{Y}$ goes to the transmitting pin (TXD). X0 and Y0 are connected to the Tx and Rx pins of the GSM module respectively. Similarly X1 and Y1 are connected to the GPS module. While enable (INH), A and $\mathrm{B}$ are at logic low, the Tx pin of the GSM module is selected. However, when enable is high, no channel is active irrespective of the logic level at A and $\mathrm{B}$. This is indicated by ' $\mathrm{x}$ ' (don't care) as shown in Table 1 . In this way, the microcontroller is able to switch communication between the two modules.

\section{PIR Sensor}

PIR sensors use the concept of Pyro electricity to detect the presence of persons. PIR are basically low cost, low power components made of sensors capable of 
developing an electric signal in response to a variation in the incident thermal radiation. It can sense the presence of human within its coverage range. The PIR was used in [31] to detect the presence/absence of home users such that all appliances automatically turns on or turns off as the case may be. In this work, it was used to detect intrusion whenever the home user is away. Thus, an alert is sent to the user in the case of intrusion detection. This feature is only active whenever the user is away from home. The PIR sensor is as shown in Fig 4.

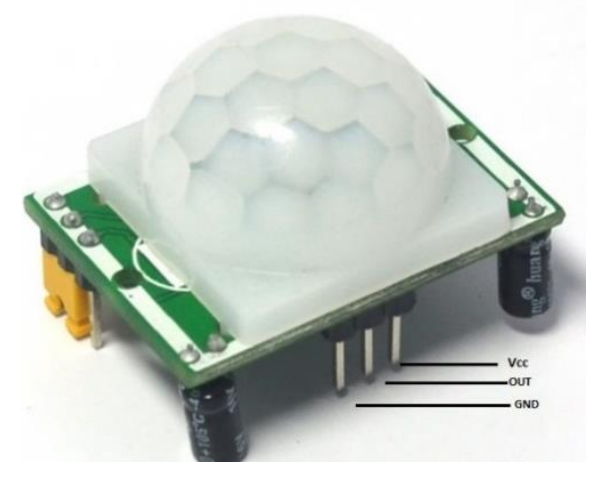

Fig.4. The PIR sensor module

\section{E. Home Automation Processing Unit}

At the heart of the home automation system is the processing unit which serves has the 'brain' of the entire system. It monitors and controls all connected modules. The central processing unit was built around an ATmega8 microcontroller which is a CMOS Reduced Instruction Set Computer (RISC). It is an 8-Bit Microcontroller, Insystem Programmable with Flash code storage, reprogrammable up to 1000 write/rewrite times. Its features includes 32 working registers, single clock cycle execution giving up to $1 \mathrm{MIPs} / \mathrm{MHz}$, 6-channel ADC with 10-bit Accuracy, 23 Programmable I/O Lines, 4.5 to $5.5 \mathrm{~V}$ operating voltage.

The processing unit, as the name suggests, is where the actions requested by users are processed. For the purposes of security, all the messages received are checked by authenticating the senders, checking the integrity of the messages to ensure they are in the required format. If the received message is intact, the processing unit effects the user's request by turning ON/OFF the required appliance(s). The Android App has been programmed to send users' command in the required format except for the case of non-smart phone users. After implementing user's request, the processing unit sends a feedback message via the GSM module to update the user about the current status of the home appliances.

\section{F. Appliance Control and Feedback Unit}

The current transformer is an electric circuit mostly used for measuring and evaluating electricity consumption of particular appliances. However, in this paper, we have deployed it as a current sensor to detect flow of electrical current from the relay circuit to the connected appliances. The output of the current sensor was connected to the ADC (analog to digital converter) port pin of the microcontroller. Fig. 5, shows the circuit connection of the current transformer (CT) to a relay and the connected load.

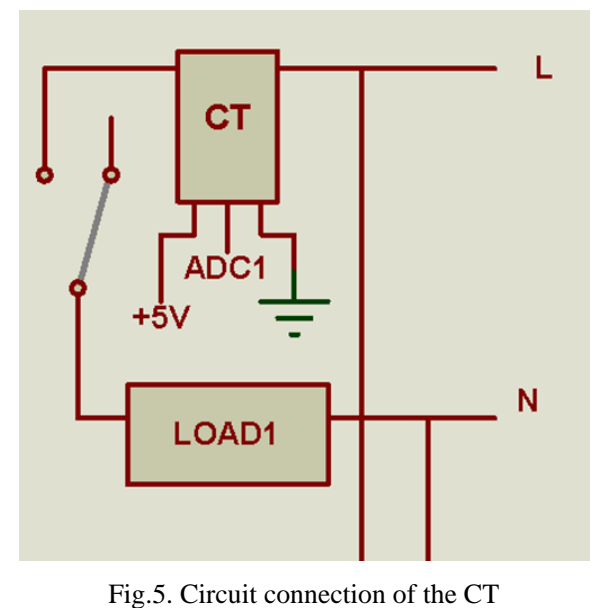

$\mathrm{L}$ and $\mathrm{N}$ indicates the live and neutral from the mains supply respectively, which can be $220 \mathrm{~V}$ or $110 \mathrm{~V}$ depending on the requirement of the appliance. The CT contains a current to voltage converter such that the equivalent current flowing in the appliance is read as voltage. The output of the CT is connected to channel 1 of the microcontroller ADC. Whenever the microcontroller activates an appliance, it monitors the corresponding ADC channel to ensure flow of current before sending feedback to the user. To avoid false alarm, several samples were taken and the average value is obtained as in

$$
V_{s}=\frac{V_{s 1}+V_{s 2}+\ldots+V_{s n}}{n}
$$

Where $V_{s}$ is the equivalent current flowing through the appliance read as voltage, $\mathrm{n}$ is the number of samples and $V_{s n}$ are the various samples.

\section{SYSTEM IMPLEMENTATION}

This section discusses the implementation of the proposed system. The implementation is divided into hardware, firmware, and Android App implementations.

\section{A. Hardware Implementation}

The implementation of the proposed system is illustrated in Fig. 6. It shows the connection of the GSM module, which serves as the SMS gateway, the Bluetooth module, PIR sensor, LCD, and relays to the microcontroller. The system is powered from the home $220 \mathrm{~V}$ mains supply. A $12 \mathrm{~V}$ battery was also used as backup to keep the system active in the case of power cut.

The operation of the hardware solely depends on the program code running on the microcontroller chip. The program was written using the Atmel Studio 6 IDE. Detailed implementation of this program will be discuss 
shortly. The LCD displays the various status of the system.

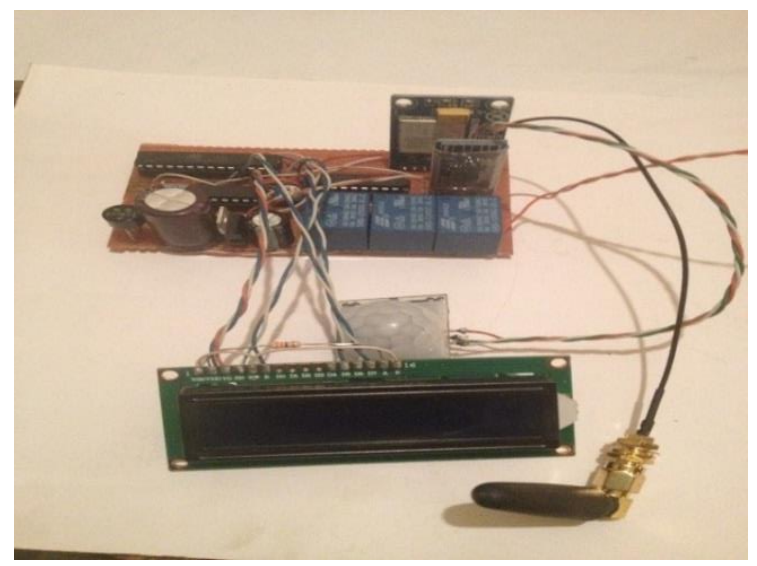

Fig.6. System implementation

The output of the mains power supply, regulated to $5 \mathrm{~V}$, is connected to channel 0 of the microcontroller ADC. This enables the processing unit to detect power cut and update the user on request.

\section{B. Firmware Implementation}

Firmware code refers to the program running on the microcontroller chip. The program was written and debugged using $\mathrm{C}++$ language from the Atmel studio 6 IDE which is a free open source software. The flowchart of the program is shown in Fig. 7.

After initializing the modules, the microcontroller monitors the GSM and Bluetooth modules to receive users' request. Both modules use the RS232 serial communication protocol. However, the GSM module requires the AT command set to carry out specific function. The following are some of the major AT commands used in this work.

\section{$>\mathrm{AT}+\mathrm{CMGR}=1$ reads message number 1 \\ $>\mathrm{AT}+\mathrm{CMGD}=1$ delete the SMS \\ $>\mathrm{AT}+\mathrm{CSMS}$ selects message service}

On receiving a message the content of the message is copied, verified for user authenticity and accurate control sequence. If the message is from an unauthorized user or not well formatted, it is discarded and no action is taken by the processing unit. A well formatted message received from an authorized user is decoded to determine the user's request. A switch...case command was used to achieve this function. Depending on the command received, specific instructions were written for the different case. The program was built to generate the Hex file and transferred to the microcontroller chip using a universal chip programmer.

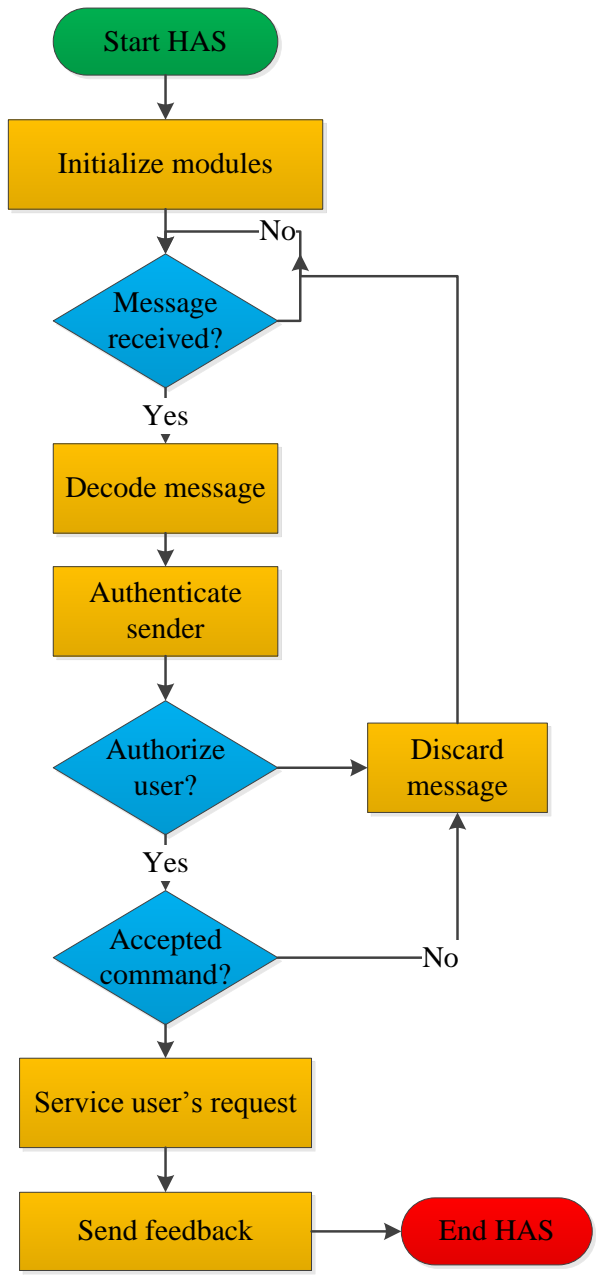

Fig.7. Program flowchart

\section{User Friendly Interface}

This involves development of a smart phone based application used to control the connected home appliances either through Bluetooth or SMS. The application serves as an interface by which the user can control the appliances conveniently without the need to memorize control commands. The mobile App runs on an Android OS. This was selected due to its widespread among consumers.

The App was developed using Java programming language from the Eclipse IDE. Eclipse IDE is mostly primarily used for the development of java applications, however, it can also be used to develop applications in other high level languages by using plugins such as: $\mathrm{C}++$, Python, R, and Ruby. The App interface is as shown in Fig. 8. With a simple click, the user can switch between using Bluetooth or SMS to control the home appliances. 


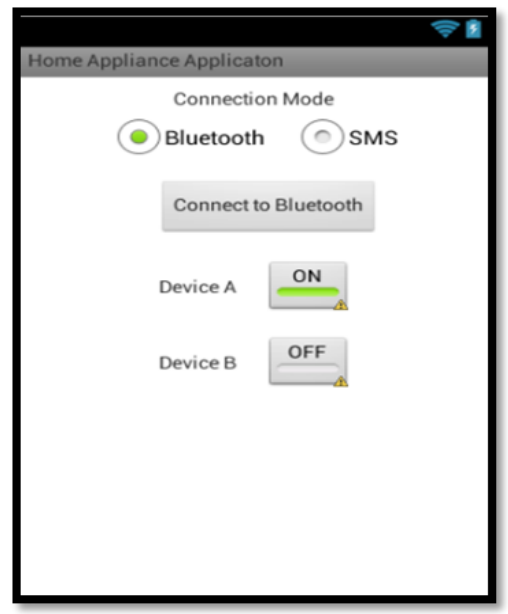

Fig.8. Prototype GUI of the Android phone application

The following simple steps are required to use the App:

$\checkmark \quad$ Install App on smart phone running Android OS

$\checkmark$ Launch App

$\checkmark$ Select Bluetooth or SMS control

$\checkmark$ For Bluetooth control, click on connect Bluetooth

$\checkmark$ Select device to turn ON/OFF

$\checkmark \quad$ Wait for feedback

\section{PERFormanCE EVALUATION}

To evaluate effectiveness of the developed system, two different devices (bulb and pumping machine switch) were connected to it. The developed Android App was also installed on a smart phone running Android OS. There are two mode of operation of the HAS: normal mode and away mode. In normal mode, PIR sensor is inactive, therefore intrusion is not detected in this mode. Away mode can be activated by turning ON a switch placed on the system or by sending an SMS to the HAS in case where the user forget to turn ON the switch. In this mode, the HAS can detect intruders and send feedback to the user.

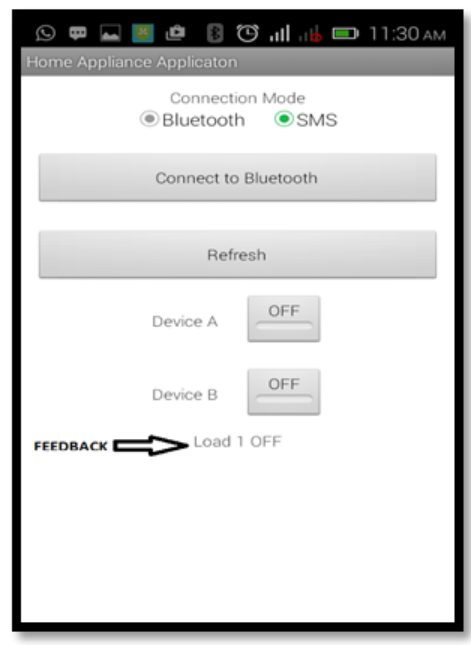

Fig.9. Snapshot showing the feedback message received when Load 1 was switched OFF.
Fig. 9, shows the user interface in the SMS mode. To control an appliance, the user simply toggles the switch. Fig. 9, shows the case when appliance 1 (Device A) is switched OFF. It also shows the feedback received after the appliance was switched OFF. Similarly, Fig. 10, shows when the appliance is switched ON.

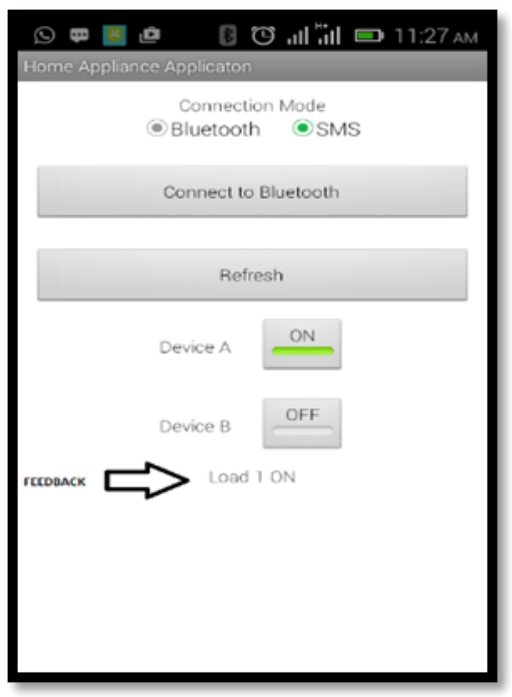

Fig.10. Snapshot showing Load 1 switched ON and the feedback received.

Similar results were obtained using the Bluetooth mode. On every control (ON/OFF) using the Bluetooth mode, the user saves 44.00 which is equivalent to $\$ 0.0127$. The PIR was tested by human movement around its coverage range while the away mode is active. Fig. 11, shows the SMS received by the user indicating detection of an intruder.

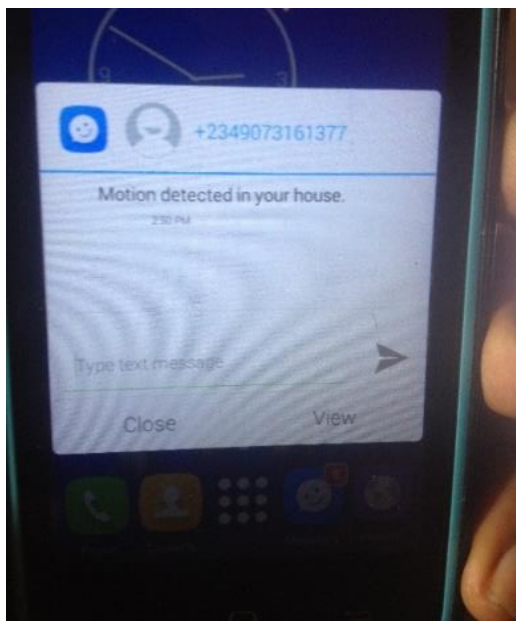

Fig.11. Snapshot showing SMS received on detection of an intruder.

\section{CONCLUSION}

In this paper a home automation system using two widely used communication technology, Bluetooth and GSM have been presented. An ideal smart-home system should be affordable, reliable, easy to use or control and 
provides accurate and real time feedback. The proposed system is affordable and has low running cost through the implementation of both Bluetooth and GSM control. Consumers neither require internet connection nor a computer to make use of the proposed system. The system has a friendly interface through the use of a simplified Android application and provides accurate and real time feedback during Control. In addition, it also provides a security feature to help detect intrusion or unauthorized entry while the home user is away.

\section{REFERENCES}

[1] M. Yan and H. Shi, "Smart Living Using Blueooth-Based Android Smartphone," Int. J. Wirel. Mob. Networks, vol. 5, no. 1, pp. 65-72, 2013.

[2] R. Piyare and M. Tazil, "Bluetooth based home automation system using cell phone," in 2011 IEEE 15th International Symposium on Consumer Electronics (ISCE), 2011, pp. 192-195.

[3] S. Anwaarullah and S. V Altaf, "RTOS based Home Automation System using Android," Int. J. Adv. Trends Comput. Sci. Eng., vol. 2, no. 1, pp. 480-484, 2013.

[4] K. Gill, S. Yang, F. Yao, and X. Lu, "A ZigBee-Based Home Automation System," IEEE Trans. Consum. Electron., vol. 55, no. 2, pp. 422-430, 2009.

[5] S. Kanagamalliga, S. Vasuki, A. V. Priya, and V. Viji, "A Zigbee and Embedded based Security Monitoring and Control System," Int. J. Inf. Sci. Tech., vol. 4, no. 3, pp. 173-178, 2014.

[6] J. Zhu, X. Gao, Y. Yang, H. Li, Z. Ai, and X. Cui, "Developing a voice control system for zigbee-based home automation networks," in 2010 2nd IEEE InternationalConference on Network Infrastructure and Digital Content, 2010, pp. 737-741.

[7] Y. B. Krishna and S. Nagendram, "Zigbee based voice control system for smart home," Int. J. Comput. Technol. Appl., vol. 3, no. 1, pp. 163-168, 2012.

[8] M. A. Samad and Mv. CHARY, "Design of Remote Intelligent Smart Home System Based on Zigbee and GSM Technology,” Int. J. Eng. Trends Technol. (IJETT)Volume, vol. 4, 2013.

[9] K. Baraka, M. Ghobril, S. Malek, R. Kanj, and A. Kayssi, "Low cost arduino/android-based energy-efficient home automation system with smart task scheduling," in Proceedings - 5th International Conference on Computational Intelligence, Communication Systems, and Networks, CICSyN 2013, 2013, pp. 296-301.

[10] A. Alheraish, "Design and implementation of home automation system," Consum. Electron. IEEE Trans., vol. 50, no. 4, pp. 1087-1092, 2004.

[11] W. Mohd Helmy Abd, A. Norzilawati, J. Ayob, and A. K. Herdawatie, "GSM Based Electrical Control System for Smart Home Application,” J. Converg. Inf. Technol., vol. 5, no. 1, pp. 33-39, 2010.

[12] L. S. L. Siwen and L. Y. L. Yunhong, "Design and Implementation of Home Automation System," in 2008 International Symposium on Information Science and Engineering, 2004, vol. 2, no. 4, pp. 1087-1092.

[13] S. Das, N. Debabhuti, R. Das, S. Dutta, and A. Ghosh, "Embedded system for home automation using SMS," in 1st International Conference on Automation, Control, Energy and Systems - 2014, ACES 2014, 2014.

[14] C. Felix and I. Jacob Raglend, "Home automation using
GSM," in 2011 International Conference on Signal Processing, Communication, Computing and Networking Technologies, 2011, pp. 15-19.

[15] M. shaker Nasr and F. H. A. sale. Azwai, "Friendly home automation system using cell phone and J2ME with feedback instant voice messages," in 2009 IEEE/ACS International Conference on Computer Systems and Applications, 2009, pp. 531-538.

[16] R. Teymourzadeh, S. A. Ahmed, K. W. Chan, and M. V. Hoong, "Smart GSM based home automation system," in Proceedings - 2013 IEEE Conference on Systems, Process and Control, ICSPC 2013, 2013, pp. 306-309.

[17] M. Van Der Werff, X. Gui, and W. L. Xu, "A mobilebased home automation system," in 2005 2nd Asia Pacific Conference on Mobile Technology, Applications and Systems, 2005, p. 5 pp.-pp.5.

[18] Y. Zhao and Z. Ye, "A low cost GSM/GPRS based wireless home security system," IEEE Trans. Consum. Electron., vol. 54, no. 2, pp. 567-572, 2008.

[19] T. Ahammed and P. P. Banik, "Home Appliances Control Using Mobile Phone," in Proceedings of 2015 3rd International Conference on Advances in Electrical Engineering 17-19 December, 2015., 2015, pp. 251-254.

[20] A. Mulla, J. Baviskar, and A. Baviskar, "DTMF Based Automation System with Reduction of Noise Using Goertzel DFT Estimation,” in 2014 Fourth International Conference on Communication Systems and Network Technologies, 2014, pp. 1124-1129.

[21] T. M. Ladwa, S. M. Ladwa, R. S. Kaarthik, A. R. Dhara, and N. Dalei, "Control of remote domestic system using DTMF," in International Conference on Instrumentation, Communication, Information Technology, and Biomedical Engineering 2009, 2009, pp. 1-6.

[22] A. Z. Alkar, J. Roach, and D. Baysal, "IP based home automation system," IEEE Trans. Consum. Electron., vol. 56, no. 4, pp. 2201-2207, 2010.

[23] S. C. Son, N. W. Kim, B. T. Lee, C. H. Cho, and J. W. Chong, "A time synchronization technique for coap-based home automation systems," IEEE Trans. Consum. Electron., vol. 62, no. 1, pp. 10-16, 2016.

[24] A. Z. Alkar and U. Buhur, "An internet based wireless home automation system for multifunctional devices," IEEE Trans. Consum. Electron., vol. 51, no. 4, pp. 1169$1173,2005$.

[25] S. Manohar and D. M. Kumar, "E-mail Interactive Home Automation System," Int. J. Comput. Sci. Mob. Comput., vol. 4, no. 7, pp. 78-87, 2015.

[26] S. Jain, A. Vaibhav, and L. Goyal, "Raspberry Pi based interactive home automation system through E-mail," in ICROIT 2014 - Proceedings of the 2014 International Conference on Reliability, Optimization and Information Technology, 2014, pp. 277-280.

[27] H. Jinsoo, Y. Jaekwan, J. Jonghyun, and P. Kwang-Roh, "User-friendly home automation based on 3D virtual world," Consum. Electron. IEEE Trans., vol. 56, no. 3, pp 1843-1847, 2010.

[28] V. Madan and S. R. N. Reddy, "GSM-Bluetooth based remote monitoring and control system with automatic light controller," Int. J. Comput. Appl., vol. 46, no. 1, pp. 20-28, 2012.

[29] N. M. Morshed, G. M. Muid-Ur-Rahman, M. R. Karim, and H. U. Zaman, "Microcontroller based home automation system using Bluetooth, GSM, Wi-Fi and DTMF," in Advances in Electrical Engineering (ICAEE), 2015 International Conference on, 2015, pp. 101-104. 


\section{Authors' Profiles}

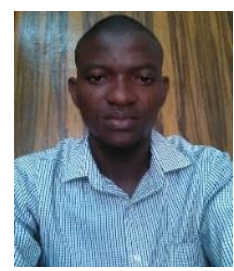

Salihu O. Aliyu is a lecturer at the Department of Telecommunication Engineering, Federal University of Technology, Minna Nigeria. He received the BEng. degree in electrical and computer engineering at Federal University of Technology, Minna in 2010 and M.Sc. degree in electrical engineering from the King Fahd University of Petroleum and Minerals, Dhahran, KSA in 2015.

From 2011 to 2012, he was a Research Assistant with Manhenrycee Electronics Construction Company, Ilorin Nigeria His research interests include digital signal/image processing, embedded/intelligent system design, pattern recognition and smart grid.

He was a recipient of the best paper award in 2016, at the $13^{\text {th }}$ IEEE International Multi-Conference on Systems, Signals and Devices 2016 Leipzig, Germany.

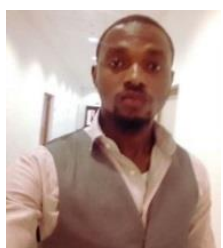

Abdulazeez Yusuf had his Bachelor of Engineering in Telecommunication Engineering from the Department of Telecommunication Engineering, Federal University of Technology, Minna, Niger State, Nigeria in 2016. He is a promising embedded system specialist. His areas of research includes embedded, and Intelligent Systems design.

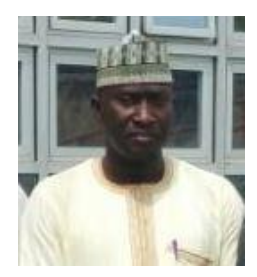

Umar Abdullahi received the B.Eng in Electrical/Computer Engineering and M.Eng in Communications Engineering both from Federal University of Technology, Minna, Niger State, Nigeria in 2008 and 2014 respectively. $\mathrm{He}$ is an Assistant Lecturer with the Department of Telecommunications Engineering, Federal University of Technology, Minna, Niger State. His research interest includes Radio Resource Management in WiMAX network, Energy Management in Wireless Sensor Network (WSN), Internet-of- Things (IoT) and Home Automations. Mr. Abdullahi's awards include the 2009 Central Bank of Nigeria award and NYSC Venture Price award. Contact him at umarabdullahi@futminna.edu.ng.

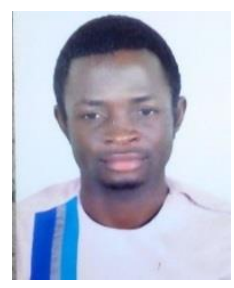

Mustapha Hafiz had his Bachelor of Engineering in electrical and computer engineering from the Department of electrical and computer engineering, Federal University of Technology, Minna, Niger State, Nigeria in 2010. His research interest includes embedded/intelligent system design. $\mathrm{He}$ is currently with synchronous logic engineering limited, minna Nigeria. Contact him at bola4micro@gmail.com.

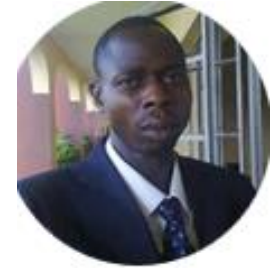

AJAO, Lukman Adewale is a Senior Technological Researcher, $\mathrm{He}$ is currently working in the Department of Computer Engineering, Federal University of Technology, Minna, Nigeria. He obtained Postgraduate Diploma in Computer Science (PGDCS) in 2013 from University of Ilorin, Ilorin, Nigeria. He is currently a Master of Engineering research student in the Department of Computer Engineering, Federal University of Technology, Minna. The research interest are Embedded System based Internet of Things, Wireless Sensor Network, FPGA and Networking.

How to cite this paper: Salihu Aliyu, Abdulazeez Yusuf, Umar Abdullahi, Mustapha Hafiz, Lukman A. Ajao,"Development of a Low-Cost GSM-Bluetooth Home Automation System", International Journal of Intelligent Systems and Applications(IJISA), Vol.9, No.8, pp.41-50, 2017. DOI: 10.5815/ijisa.2017.08.05 